\title{
Morfología del polen de Heliconia spp. (Heliconiaceae) de México y su relación interespecífica e intraespecífica
}

\author{
Simitrio Ortiz-Curiel ${ }^{1}$, Guillermo López-Guillén ${ }^{1}$, Carlos-Hugo Avendaño-Arrazate ${ }^{1}$ \\ $\&$ Misael Martínez-Bolaños ${ }^{1}$ \\ 1. Instituto Nacional de Investigaciones Forestales Agrícolas y Pecuarias, Campo Experimental Rosario Izapa, Carretera \\ Tapachula-Cacahoatán km 18, Tuxtla Chico, Chiapas, México; ortiz.simitrio@inifap.gob.mx, \\ lopez.guillermo@inifap.gob.mx, avendano.carlos@inifap.gob.mx,martinez.misael@inifap.gob.mx
}

Recibido 28-II-2020. Corregido 03-V-2020. Aceptado 22-VI-2020.

\begin{abstract}
Pollen morphology of Heliconia spp. (Heliconiaceae) from Mexico and its interspecific and intraspecific relationship. Introduction: Knowledge about palynology in the Heliconia genus basically concerns species from Central and South America, while studies of species in the Mexican Neotropic are limited. Objective: To contribute to the palynological knowledge and understand the morphological interrelation of pollen from the Heliconia genus of the Mexican Neotropic, as part of reproductive biology studies that may contribute in future works of genetic improvement. Methods: The study was conducted in nine species (12 accessions) of Heliconia native to Mexico which are found in the Germplasm Bank of the National Institute of Agricultural and Livestock Forestry Research (INIFAP). Floral pollen was collected in anthesis and stored for $24 \mathrm{~h}$ in $2 \%$ glutaraldehyde and $50 \%$ ethanol, then washed in $50 \%$ ethanol and dehydrated in 70, 90 and 100 $\%$ alcohol for $30 \mathrm{~min}$. The samples were left to dry at the critical point of the $\mathrm{CO}_{2}$, mounted in aluminum cylinders covered with double conductive adhesive carbon tape and analyzed with a layer of approximately $20 \mathrm{~nm}$ gold-palladium. Samples were observed in MEB model TOPCON, SM-510. Results: Pollen measures, 50.22 $\mu \mathrm{m}$ to $70.56 \mu \mathrm{m}$, is heteropolar and has an euoblate, suboblate or oblate-spheroidal shape. Distal and proximal faces presented different degrees of convexity; both with micro-echinate or micro-verrucate ornamentation. Morphological characteristics, pollen shape, shape of the proximal and distal faces, as well as ornamentation, showed some homogeneity. However, five groups were formed through the analysis of hierarchical grouping $\left(\mathrm{R}^{2}=0.05\right)$. Group I present some micro-perforations in the tectum, high density of micro-echinates and high convexity of the proximal face. In contrast to Group I, Group II has their distal face convex with half density of micro-echinates and a plane-subconvex proximal face. Group III is represented by H. latispatha and group IV is represented by H. librata, both groups have small pollen (for the present study). Although, H. latispatha has a verrucae distal face and a plane proximal face, germinating pore that is distinctively protruding, and $H$. librata has verrucae in both faces. In group $\mathrm{V}, \mathrm{H}$. collinsiana has a distinctively protruding germinating pore and a "corrugated circle-shaped" in the proximal face. Among varieties, H. collinsiana is $10 \mu \mathrm{m}$ higher than $H$. collinsiana var. velutina in its equatorial plane. Conclusions: Heterogeneity of characteristics allowed pollen groups to be generated; whilst relatively homogeneous characteristics allowed for recognition of species and varieties within the groups. The morphology of the Heliconia species in Mexico confirms the previously described characteristics; however, additional features such as the presence of micropores and the corrugated circle-shaped in the proximal face stand out.
\end{abstract}

Key words: palynology; Heliconia; endemic; diversity; neotropical.

Ortiz-Curiel, S., López-Guillén, G., Avendaño-Arrazate, C.H., \& Martínez-Bolaños, M. (2020). Morfología del polen de Heliconia spp. (Heliconiaceae) de México y su relación interespecífica e intraespecífica. Revista de Biología Tropical, 68(3), 933-946. 
El género Heliconia L. (Heliconiaceae) está integrado por un grupo de aproximadamente 220 especies (Kress, Betancur, \& Echeverry, 1999), las cuales crecen de manera natural desde el norte de México hasta el sur de Brasil incluyendo las islas del Caribe (Berry \& Kress, 1991) e islas en la región de Indonesia en el Océano Pacífico (Kress, 1990). México posee al menos 14 especies nativas las cuales habitan en selvas y bosques (Ferreira de Castro, May, \& Gonçalves, 2007), en suelos ácidos a alcalinos y desde los 36 hasta los 1 700 msnm (Ortiz-Curiel et al., 2015). Entre las especies nativas existen endémicas como H. uxpanapensis Gutiérrez Báez (GutiérrezBáez, 1987) y $H$. veracruzensis Gutiérrez Báez (Gutiérrez-Báez, Avendaño-Reyes, \& ZamoraCrescencio, 2016).

La similitud morfológica que muchas especies del género Heliconia comparten en su fase juvenil y lo contrastante que son en la etapa reproductiva por las formas raras y colores de su inflorescencia, son motivo de atención de coleccionistas, viveristas y ambientalistas. En el aspecto científico, la biología y sus distintas disciplinas se han enfocado a conocer la diversidad genética (Sheela, Geetha-Lekshmi, Jayachandran-Nair, \& Rajmohan, 2006), su genética poblacional (Suárez-Montes, Fornoni, \& Nunez-Farfán, 2011), su biología reproductiva (Bruna, Kress, Marques, \& Da Silva, 2004) y estudios de interacción con sus polinizadores (Betts, Hadley, \& Kress, 2015). De manera complementaria, disciplinas como la palinología han contribuido en aspectos de ontogenia y filogenia (Kress \& Stone, 1982, 1983) de sistemática y (Kress, 1990) y taxonomía (Kress \& Stone 1983).

Kress \& Stone (1983), en su estudio palinológico de 27 especies de Heliconia que tienen como origen zonas de Centroamérica y Sudamérica, encontraron que el polen es heteropolar, subisopolar o isopolar y que la variación que existe en su morfología esporófita (con inflorescencia erecta versus inflorescencia colgante) no siempre es en la misma magnitud en su morfología polínica, ya que en ambos tipos de inflorescencias el polen puede ser isopolar o subisopolar o con forma euoblato a suboblato. Pese a lo anterior, los mismos autores indicaron que a nivel intraespecífico, la forma y la longitud del eje ecuatorial de los granos de polen son consistentes y que dichas características, además de la ornamentación de la exina, permiten clasificar variedades de Heliconia.

Respecto a los morfismos que describieron Kress (1990) en las especies de Heliconia del Pacífico (endémicas), el polen es de forma oblato-esferoidal únicamente y su ornamentación es ligeramente verrugada o psilada, tanto en especies con inflorescencia colgante o erecta. Mientras que Simão, Scatena, \& Bouman (2007) en una especie de Brasil, encontraron variaciones morfológicas en la vista ecuatorial del polen. Por lo anterior, la diversidad morfológica del polen obedece a la diversidad genética pero algunos morfismos del polen son propios de ciertas zonas geográficas.

A pesar de que los estudios palinológicos en el género Heliconia se iniciaron desde hace aproximadamente cuatro décadas, las especies estudiadas representan apenas el $17 \%$, y de éste, en un porcentaje menor se han estudiado las características de la exina. Aunado a esto, se han omitido a las especies que habitan de manera natural en el Neotrópico mexicano, incluyendo sus especies endémicas. En este sentido, es necesario continuar con estudios de palinología en este género, con la finalidad de conocer la morfología y con ello contribuir al conocimiento palinológico. Además, el comparativo palinológico entre especies y variedades puede mostrar aquellas características relacionadas con la autoincompatibilidad, barrera fisiológica presente en este género.

\section{MATERIALES Y MÉTODOS}

Material biológico: Se estudió el polen de nueve especies de Heliconia (12 accesiones) que están resguardadas en el Banco de Germoplasma de Heliconias del Campo Experimental Rosario Izapa del Instituto Nacional de Investigaciones Forestales, Agrícolas y Pecuarias (INIFAP). El origen, hábitat y características de las accesiones se presentan en el Tabla 1 . 
TABLA 1

Accesiones de Heliconia spp. con origen de distintas localidades de México y establecidas en el Banco de Germoplasma de Heliconias del Campo Experimental Rosario Izapa del INIFAP

TABLE 1

Accessions of Heliconia spp. originating from different locations in Mexico and established in the Heliconias Germplasm Bank of the Rosario Izapa Experimental Field of INIFAP

\begin{tabular}{|c|c|c|c|c|c|c|c|c|}
\hline Especie & $\begin{array}{l}\text { Código de } \\
\text { la accesión }\end{array}$ & $\begin{array}{c}\text { Color de } \\
\text { inflorescencia }\end{array}$ & Status & $\begin{array}{c}\text { Tipo de } \\
\text { inflorescencia }\end{array}$ & Localidad & $\begin{array}{l}\text { Altitud } \\
(\mathrm{m})\end{array}$ & $\mathrm{N}$ & W \\
\hline $\begin{array}{l}\text { H. adflexa } \\
\text { (Griggs) Standl. }\end{array}$ & Adf-03 & Rojo & Silvestre & Erecta & $\begin{array}{l}\text { Villa Corzo, } \\
\text { Chiapas }\end{array}$ & 1700 & $16^{\circ} 8^{\prime}$ & $93^{\circ} 35^{\prime}$ \\
\hline H. bourgaeana Petersen & Bou-08 & Rojo & Silvestre & Erecta & $\begin{array}{l}\text { Tezonapa, } \\
\text { Veracruz }\end{array}$ & 635 & $18^{\circ} 38^{\prime}$ & $96^{\circ} 46^{\prime}$ \\
\hline H. champneiana Griggs & Cha-07 & $\begin{array}{c}\text { Rojo- } \\
\text { variegado }\end{array}$ & $\begin{array}{l}\text { Silvestre } \\
\text { fomentada }\end{array}$ & Erecta & $\begin{array}{l}\text { Palenque, } \\
\text { Chiapas }\end{array}$ & 309 & $17^{\circ} 23^{\prime}$ & $91^{\circ} 59^{\prime}$ \\
\hline $\begin{array}{l}\text { H. collinsiana } \\
\text { var. collinsiana }\end{array}$ & Col-27 & Rojo & Silvestre & Pendular & $\begin{array}{l}\text { Tuxtla Chico, } \\
\text { Chiapas }\end{array}$ & 402 & $14^{\circ} 57^{\prime}$ & $92^{\circ} 09^{\prime}$ \\
\hline $\begin{array}{l}\text { H. collinsiana } \\
\text { var. velutina }\end{array}$ & Col-01 & Rojo & Silvestre & Pendular & $\begin{array}{l}\text { Villa corzo, } \\
\text { Chiapas }\end{array}$ & 1264 & $16^{\circ} 13^{\prime}$ & $93^{\circ} 35^{\prime}$ \\
\hline H. latispatha Benth & Lat-04 & Amarillo & Silvestre & Erecta & $\begin{array}{l}\text { Acapetahua, } \\
\text { Chiapas }\end{array}$ & 36 & $15^{\circ} 16^{\prime}$ & $92^{\circ} 42$ \\
\hline H. librata Griggs & Lib-13 & Amarillo & Silvestre & Erecta & $\begin{array}{l}\text { Uxpanapa, } \\
\text { Veracruz }\end{array}$ & 74 & $17^{\circ} 17^{\prime}$ & $94^{\circ} 35^{\prime}$ \\
\hline H. spissa Griggs & Spi-15 & Rojo & Silvestre & Erecta & $\begin{array}{l}\text { Ocosingo, } \\
\text { Chiapas }\end{array}$ & 1193 & $16^{\circ} 58^{\prime}$ & $92^{\circ} 06^{\prime}$ \\
\hline \multirow[t]{3}{*}{$\begin{array}{l}\text { H. uxpanapensis } \\
\text { Gutiérrez Báez }\end{array}$} & Uxp-10 & Rojo & $\begin{array}{l}\text { Silvestre } \\
\text { (Endémica) }\end{array}$ & Erecta & $\begin{array}{l}\text { Uxpanapa, } \\
\text { Veracruz }\end{array}$ & 101 & $17^{\circ} 15^{\prime}$ & $94^{\circ} 22^{\prime}$ \\
\hline & Uxp-12 & Anaranjado & $\begin{array}{l}\text { Silvestre } \\
\text { (Endémica) }\end{array}$ & Erecta & $\begin{array}{l}\text { Uxpanapa, } \\
\text { Veracruz }\end{array}$ & 73 & $17^{\circ} 15^{\prime}$ & $94^{\circ} 23^{\prime}$ \\
\hline & Uxp-11 & $\begin{array}{c}\text { Rojo- } \\
\text { variegado }\end{array}$ & $\begin{array}{l}\text { Silvestre } \\
\text { (Endémica) }\end{array}$ & Erecta & $\begin{array}{l}\text { Uxpanapa, } \\
\text { Veracruz }\end{array}$ & 82 & $17^{\circ} 15^{\prime}$ & $94^{\circ} 22^{\prime}$ \\
\hline $\begin{array}{l}\text { H. vaginalis Benth. } \\
\text { subsp. Mathiasiae } \\
\text { (G. S. Daniels \& } \\
\text { F. G. Stiles) L. Anders. }\end{array}$ & Vag-18 & Rojo & Silvestre & Erecta & $\begin{array}{l}\text { Palenque, } \\
\text { Chiapas }\end{array}$ & 309 & $17^{\circ} 23^{\prime}$ & $91^{\circ} 39^{\prime}$ \\
\hline
\end{tabular}

Procesamiento de muestras para Microscopía Electrónica de Barrido (MEB): Flores abiertas se colectaron entre las seis y siete de la mañana y en laboratorio se extrajeron los granos de polen por medio de un alfiler y se depositaron en microtubos de $1.5 \mathrm{ml}$. El polen de tres a cinco anteras procedentes de flores de entre una y tres plantas se mezcló para formar una muestra. Las muestras se fijaron durante $24 \mathrm{~h}$ en solución de glutaraldehído al $2 \%$ y etanol al $50 \%$ (debido a que el polen de este género no tolera la técnica de acetólisis tradicional), posteriormente se lavaron por $5 \mathrm{~min}$ en etanol al $50 \%$ y se trasvasaron a bolsitas de papel. En seguida, los granos de polen se deshidrataron en alcohol al 70, 90 y $100 \%$ por 30 minutos en cada una de las concentraciones (Márquez-Guzmán, Wong, Pérez, López, \& Munguía, 2016). Las muestras se secaron por punto crítico con $\mathrm{CO}_{2}$ en un secador SUPPLIES modelo SPI-DRY CPD y se montaron en bases de cilindros de aluminio cubiertas con cinta de carbón conductivo doble adhesiva. Posteriormente las muestras con granos de polen se cubrieron con una capa de oro-paladio (Au-Pd) de aproximadamente $20 \mathrm{~nm}$ por medio un cobertor iónico Denton Vaccum modelo Desk II. Finalmente, los granos de polen 
se observaron en un microscopio electrónico de barrido marca TOPCON SM-510 operando a 8 Kv de aceleración.

Morfometría polínica: Se tomaron electromicrografías de los granos de polen en el plano polar distal, polar proximal y ecuatorial, así como de la ornamentación de la exina y en ellas se tomaron medidas del plano polar y ecuatorial de al menos 25 granos de polen de cada accesión con el programa de cómputo libre de Collins (2007). También se registraron características particulares del téctum (presencia de microporos y la densidad de microespinas o verrugas por $\mu \mathrm{m}^{2}$ ) y particularidades de la cara proximal. Las características morfológicas de los granos de polen como la polaridad, la forma y la ornamentación de la exina se identificaron con base a Sáenz (2004) y Halbritter et al. (2018). Las variables se interpretaron mediante el análisis de agrupamiento jerárquico (AAJ). Para el AAJ se usó el paquete estadístico SAS (2012) versión 9.3 y el algoritmo por agrupamiento jerárquico con una distancia euclidiana de 0.05 para generar un dendrograma.

\section{RESULTADOS}

El polen de las especies de Heliconia se considera grande, con dimensiones de 50.2 a $70.6 \mu \mathrm{m}$; tiene una cara distal que es subconvexa, subconvexa-subconcava, convexa-truncada o convexa y se complementa con una cara proximal plana, plana-subconcava, subconcava, subconvexa-subconcava o convexa, razón por la cual es heteropolar (Fig. 1A, 1E, 1I; Fig. 2A, 2E, 2I, Fig. 3A, 3E, 3I y Fig. 4A, 4E, 4I).

Los elementos esculturales de ambas caras, distal y proximal, son microespinas (Fig. 1B, 1C, 1D H. vaginalis, 1F, 1G, 1H H. adflexa, 1J, 1K, 1L H. spissa; Fig. 2F, 2G, 2H $H$. bourgaeana, 2J, 2K, 2L H. champneiana; Fig. 3B, 2C, 2D H. uxpanapensis-10, 3F, 3G, 3H H. uxpanapensis-12; Fig. 4F, 4G, 4H H. collinsiana var. velutina, $4 \mathrm{~J}, 4 \mathrm{~K}, 4 \mathrm{~L} H$. collinsiana var. collinsiana) y espínulas menos cónicas con base ancha o redonda por lo que se consideraron microverrugas (Fig. 2B, 2C, 2D H. librata; Fig. 3J, 3K, 3L H. uxpanapensis-11 y Fig. 4B, 4C, 4D H. latispatha). La cara proximal tiene microespínulas o microverrugas aún de menor tamaño que la cara distal y están presentes en menor cantidad, en consecuencia, la exina en algunas accesiones es psilada.

Las medidas del polen con mayor contraste en su eje ecuatorial (E) fueron $50.22 \mu \mathrm{m}$ en $H$. collinsina var. velutina y $70.56 \mu \mathrm{m}$ en $H$. vaginalis; valores que permitieron identificarlos como el más pequeño y más grande respectivamente para las especies de estudio. Respecto al eje polar (P) los valores fueron $28.48 \mu \mathrm{m}$ en $H$. librata y $48.59 \mu \mathrm{m}$ en $H$. collinsiana. Esta última variable está determinada por el grado de convexidad de las caras del polen y el tipo de poro germinativo, lo cual influye en la relación P/E. En este sentido, se obtuvo el polen con forma euoblata, suboblata y oblata esferoidal (Tabla 2).

Por otra parte, el agrupamiento jerárquico con un $\mathrm{R}^{2}$ semiparcial $=0.05$ arrojó cinco grupos principales (Fig. 5). El grupo I lo integraron $H$. vaginalis, $H$. adflexa y $H$. spissa. Este grupo se diferencia fácilmente por su exina microequinada y la alta densidad de microespinas por unidad de superficie, asimismo, presentan poros en el téctum (Fig. 1D, 1H, 1L) y tienen la cara proximal altamente convexa (medio círculo). Un segundo grupo lo integraron $H$. champneiana, $H$. bourgaeana y las tres accesiones de $H$. uxpanapensis. Este grupo se identifica porque, de manera inversa al grupo I, el polen tiene la cara distal altamente convexa, mientras que la cara proximal tiende a ser plana o subconvexa, excepto $H$. librata que tiene la cara proximal convexa como las especies del grupo I.

El grupo III lo representa $H$. latispatha; su cara distal presenta microverrugas, la zona del poro germinativo es protudente, además tiene la cara proximal plana subcóncava. En el grupo IV $H$. librata se separa porque el polen es de los de menor tamaño, su cara distal es casi plana y en ambas caras la ornamentación la constituyen microverrugas. El grupo V lo integra $H$. collinsiana con sus dos variedades 


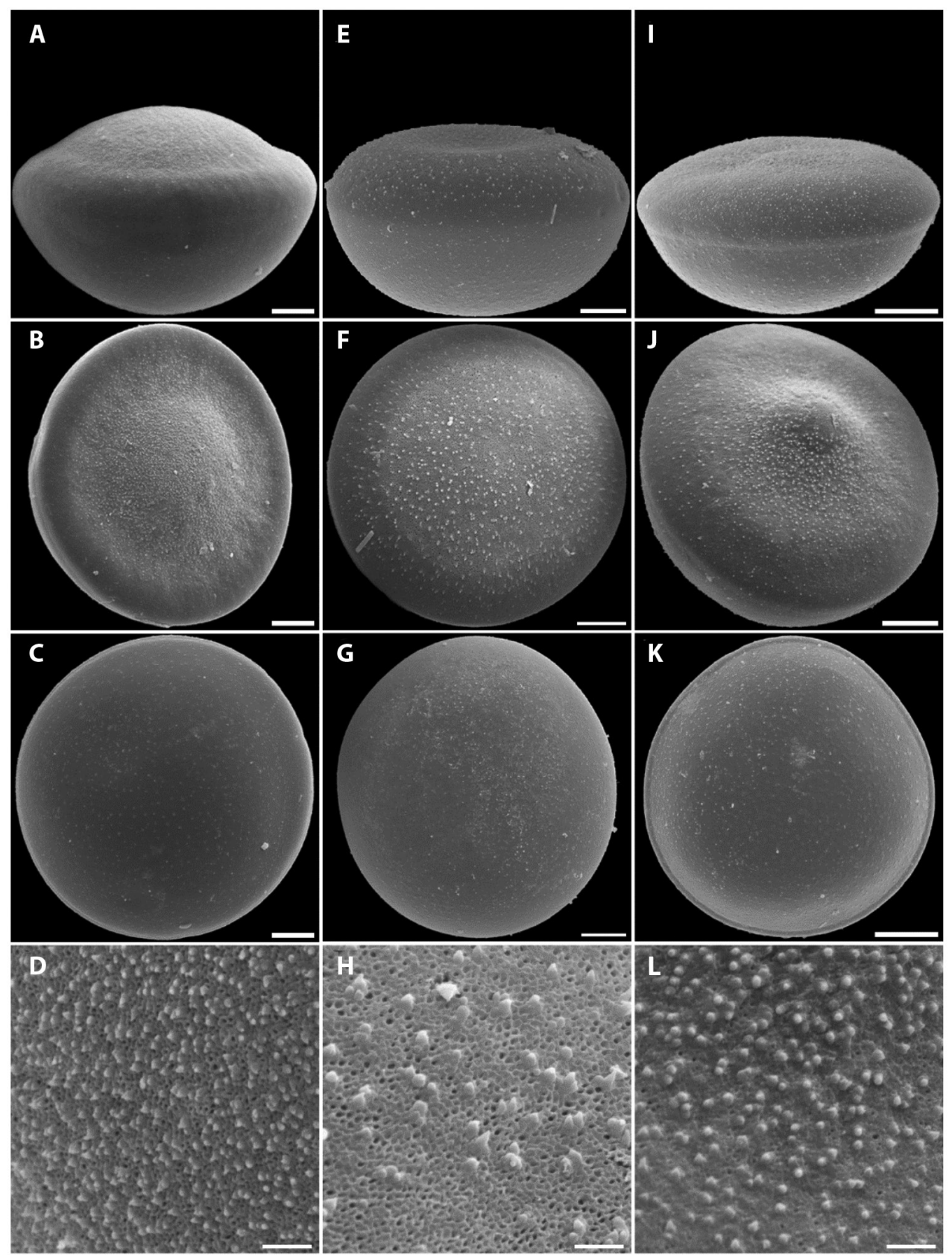

Fig. 1. (A, B, C, D) Vag-18; (E, F, G, H) Adf-03 y (I, J, K, L) Spi-15. A, E, I) en vista ecuatorial; (B, F, J) cara distal; C, $\mathbf{G}, \mathbf{K})$ cara proximal y $(\mathbf{D}, \mathbf{H}, \mathbf{L})$ ornamentación de la exina. $(\mathbf{A}, \mathbf{B}, \mathbf{C}, \mathbf{E}, \mathbf{F}, \mathbf{G}, \mathbf{I}, \mathbf{J}, \mathbf{K})$ Esc $=10 \mu \mathrm{m} ;(\mathbf{D}, \mathbf{H}, \mathbf{L})$ Esc $=2 \mu \mathrm{m}$. Fig. 1. (A, B, C, D) Vag-18; E, F, G, H) Adf-03 and (I, J, K, L) Spi-15. (A, E, I) equatorial view; (B, F, J) distal face; (C, $\mathbf{G}, \mathbf{K})$ proximal face and (D, H, L) sculpturing. (A, B, C, E, F, G, I, J, K) Esc $=10 \mu \mathrm{m}$ and $(\mathbf{D}, \mathbf{H}, \mathbf{L})$ Esc $=2 \mu \mathrm{m}$. 


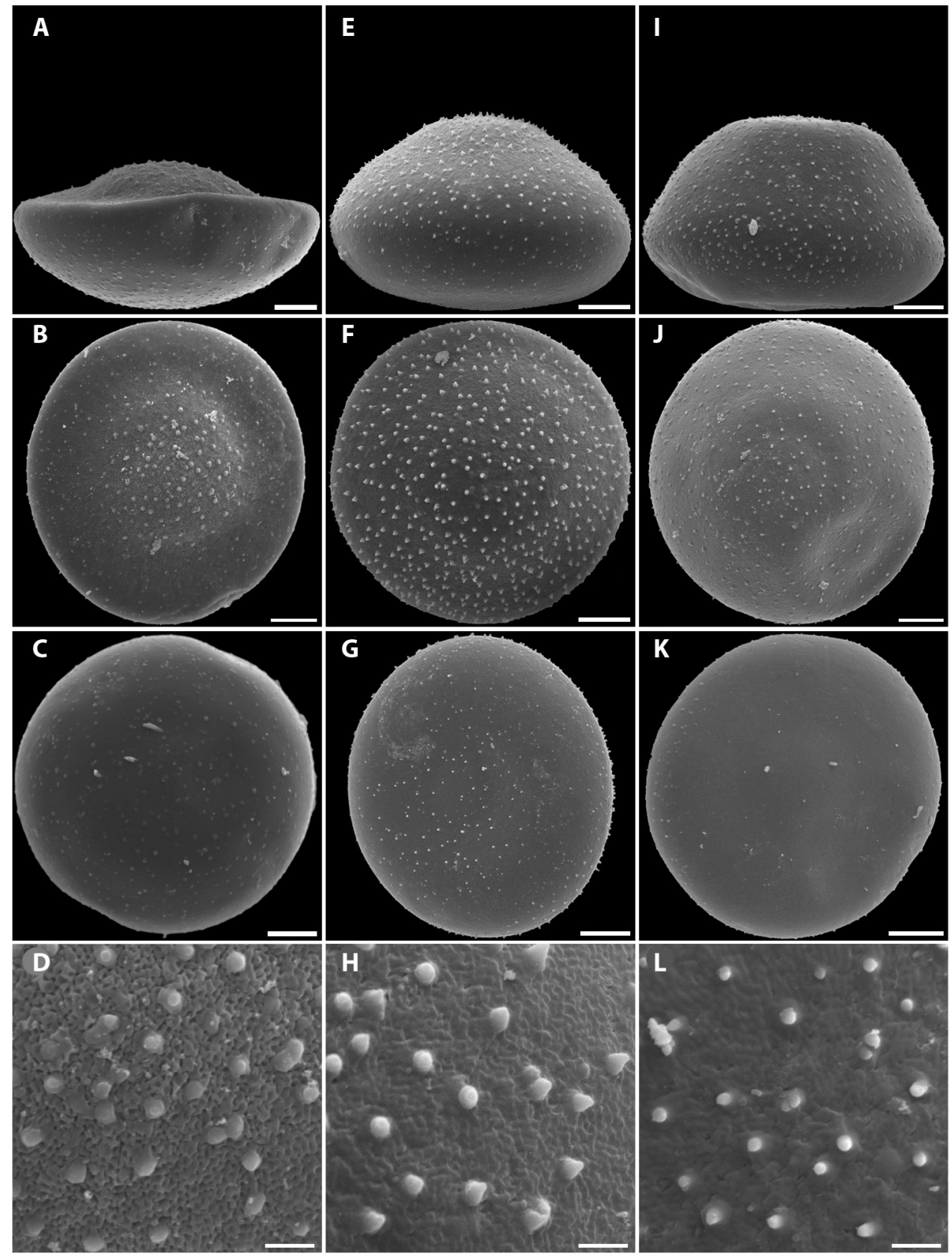

Fig. 2. (A, B, C, D) Lib-13; (E, F, G, H) Bou-08 y (I, J, K, L) Cha-07. (A, E, I) vista ecuatorial; (B, F, J) cara distal; (C, $\mathbf{G}, \mathbf{K})$ cara proximal y $(\mathbf{D}, \mathbf{H}, \mathbf{L})$ ornamentación de la exina. $(\mathbf{A}, \mathbf{B}, \mathbf{C})$ Esc $=8 \mu \mathrm{m} ;(\mathbf{E}, \mathbf{F}, \mathbf{G}, \mathbf{I}, \mathbf{J}, \mathbf{K})$ Esc $=10 \mu \mathrm{m}$ y $(\mathbf{D}$, H, L) $\mathrm{Esc}=2 \mu \mathrm{m}$.

Fig. 2. (A, B, C, D) Lib-13; (E, F, G, H) Bou-08 and (I, J, K, L) Cha-07. A, E, I) equatorial view; (B, F, J) distal face; $(\mathbf{C}, \mathbf{G}, \mathbf{K})$ proximal face and (D, H, L) sculpturing. (A, B, C) Esc $=8 \mu \mathrm{m} ; \mathbf{( E , ~ F , ~ G , ~ I , ~ J , ~ K ) ~ E s c ~}=10 \mu \mathrm{m}$ and $(\mathbf{D}, \mathbf{H}, \mathbf{L})$ $\mathrm{Esc}=2 \mu \mathrm{m}$. 


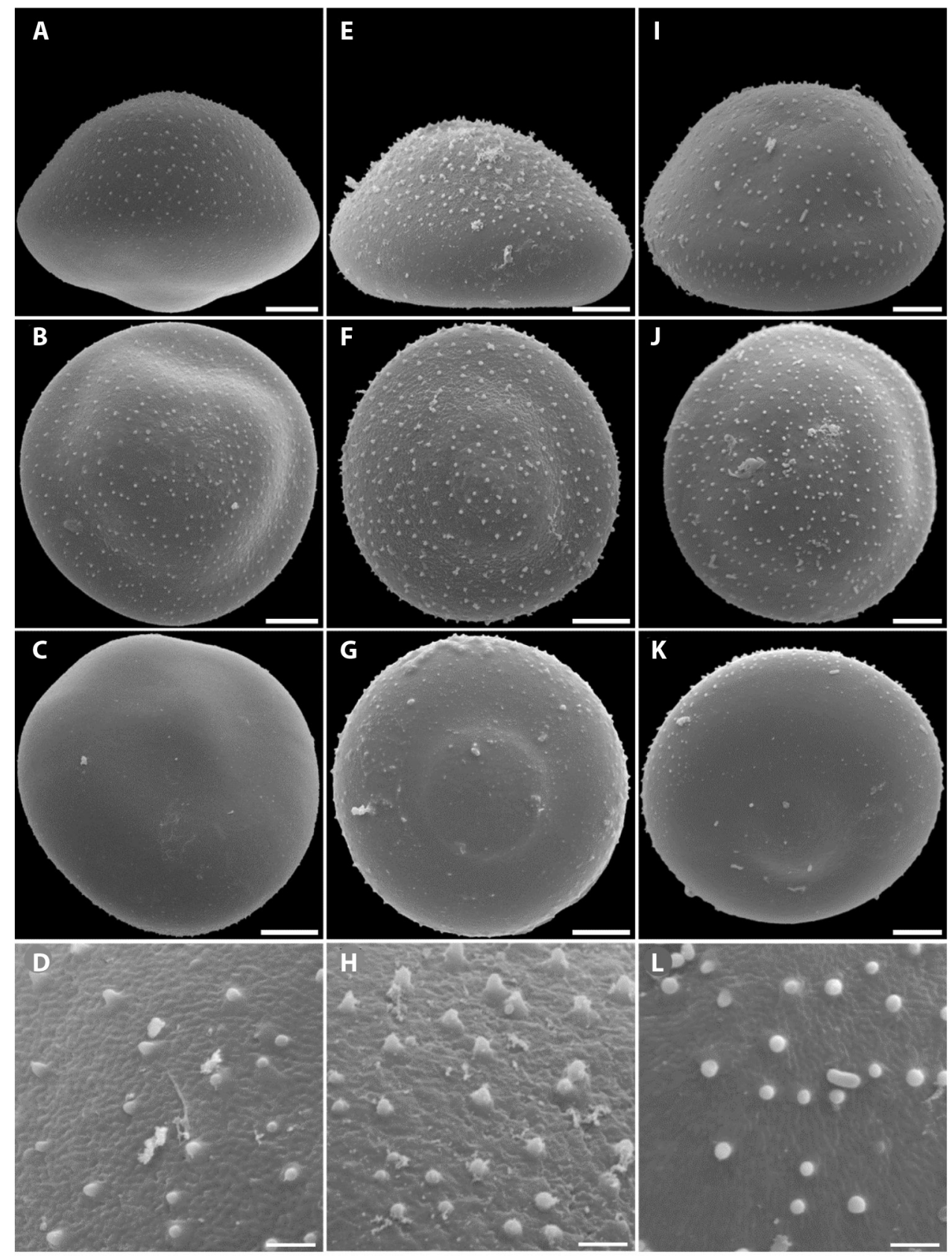

Fig. 3. (A, B, C, D) Uxp-10; (E, F, G, H) Uxp-12 y (I, J, K, L) Uxp-11. A, E, I) vista ecuatorial; (B, F, J) cara distal; (C, $\mathbf{G}, \mathbf{K})$ cara proximal y $(\mathbf{D}, \mathbf{H}, \mathbf{L})$ ornamentación de la exina. (A, B, C, E, F, G, I, J, K) Esc $=10 \mu \mathrm{m}$ y (D, H, L) Esc $=2 \mu \mathrm{m}$. Fig. 3. (A, B, C, D) Uxp-10; (E, F, G, H) Uxp-12 y (I, J, K, L) Uxp-11. A, E, I) equatorial view; (B, F, J) distal face; (C, $\mathbf{G}, \mathbf{K})$ proximal face and $\mathbf{D}, \mathbf{H}, \mathbf{L})$ sculpturing. (A, B, C, E, F, G, I, J, K) Esc $=10 \mu \mathrm{m}$ and $(\mathbf{D}, \mathbf{H}, \mathbf{L})$ Esc $=2 \mu \mathrm{m}$. 


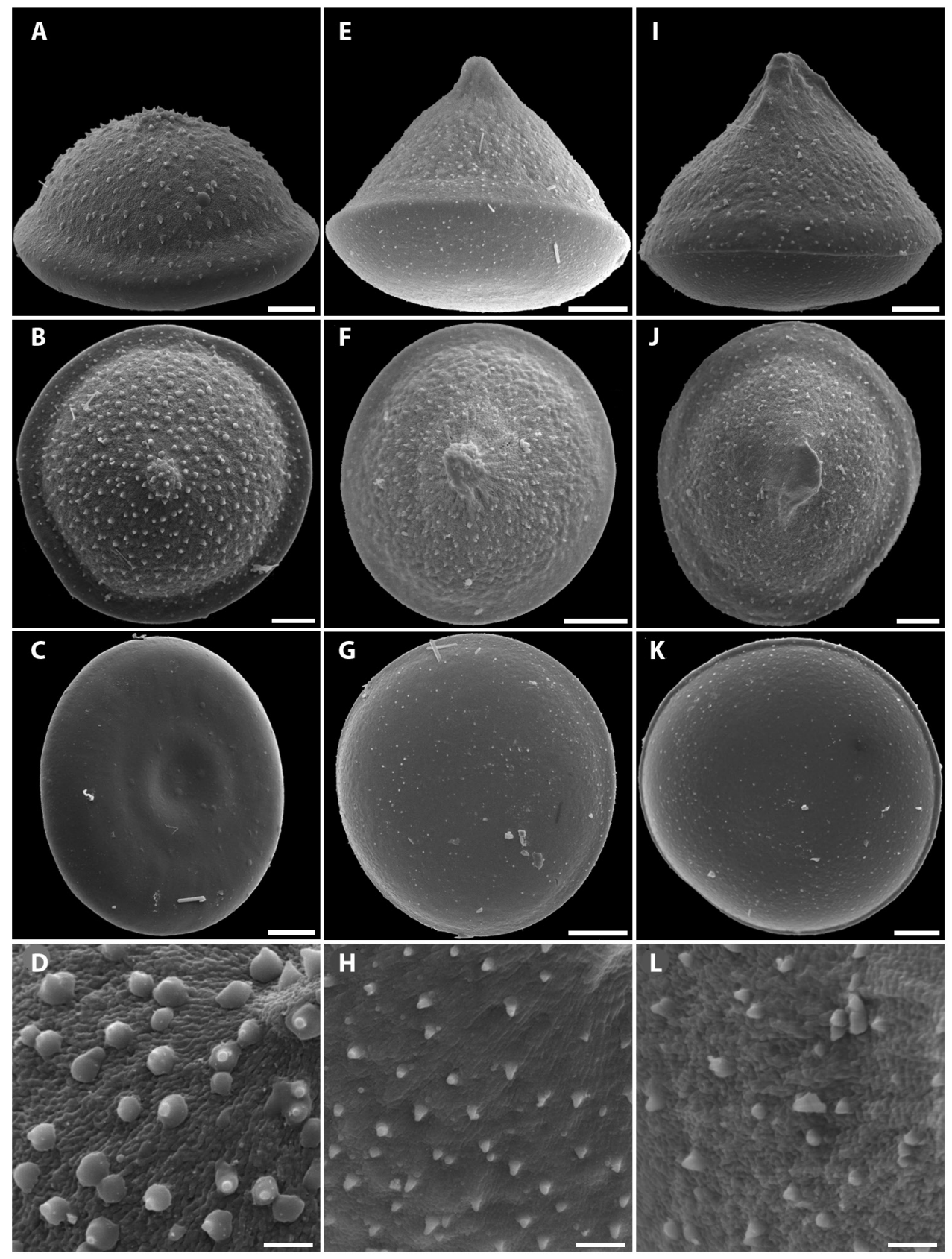

Fig. 4. (A, B, C, D) Lat-04; (E, F, G, H) Col-01 y (I, J, K, L) Col-27. (A, E, I) vista ecuatorial; (B, F, J) cara distal; (C, $\mathbf{G}, \mathbf{K})$ cara proximal y $(\mathbf{D}, \mathbf{H}, \mathbf{L})$ ornamentación de la exina. $(\mathbf{A}, \mathbf{B}, \mathbf{C}, \mathbf{E}, \mathbf{F}, \mathbf{G})$ Esc $=8 \mu \mathrm{m}, \mathbf{( I}, \mathbf{J}, \mathbf{K})$ Esc $=10 \mu \mathrm{m},(\mathbf{D}, \mathbf{H}$, L) $\mathrm{Esc}=2 \mu \mathrm{m}$.

Fig. 4. (A, B, C, D) Lat-04; (E, F, G, H) Col-01 y (I, J, K, L) Col-27. (A, E, I) equatorial view; (B, F, J) distal face; (C, G, $\mathbf{K})$ proximal face and (D, H, L) sculpturing. (A, B, C, E, F, G) Esc $=8 \mu \mathrm{m} ;(\mathbf{I}, \mathbf{J}, \mathbf{K})$ Esc $=10 \mu \mathrm{m}$ and (D, H, L) Esc $=2 \mu \mathrm{m}$. 


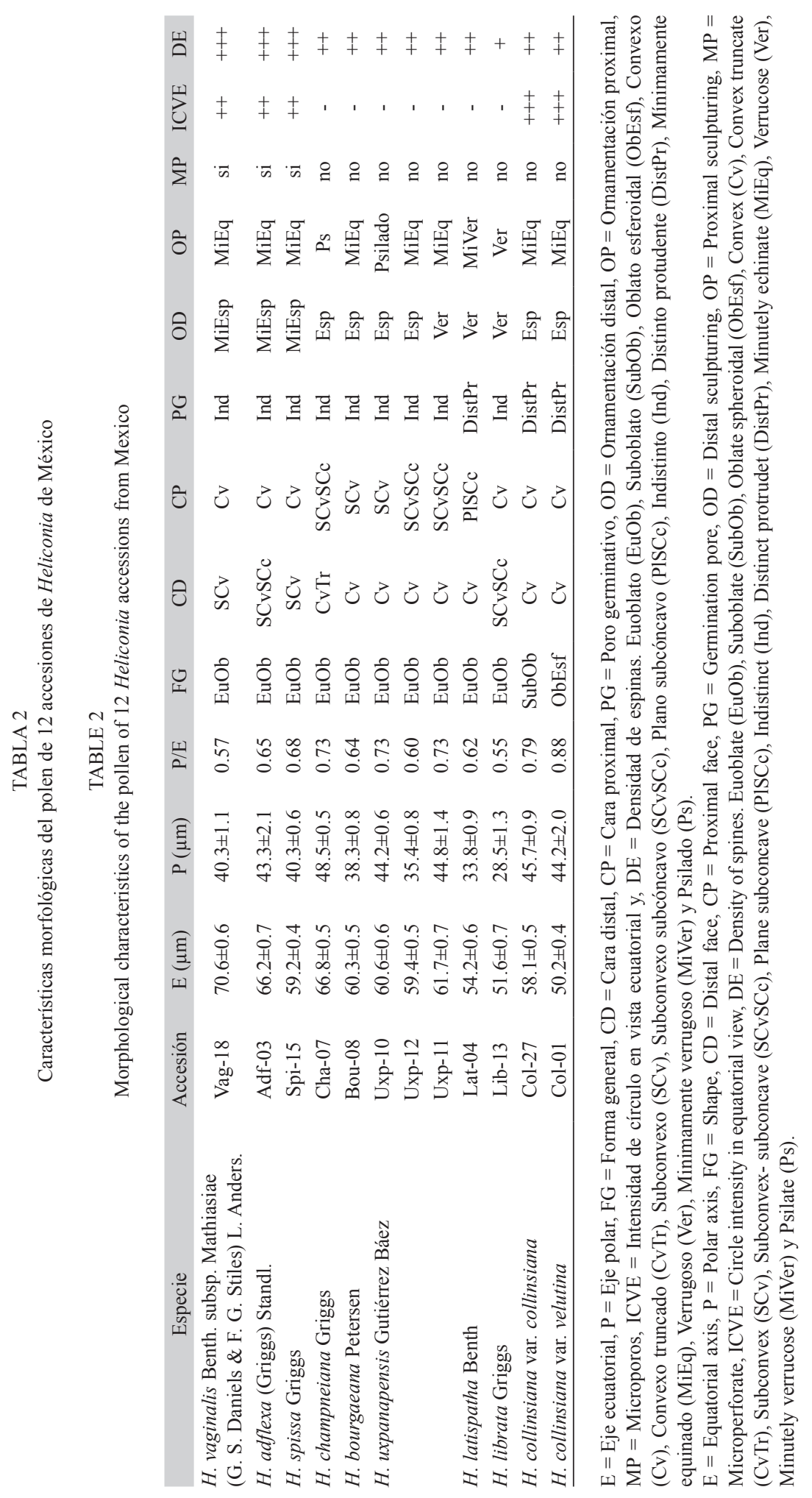




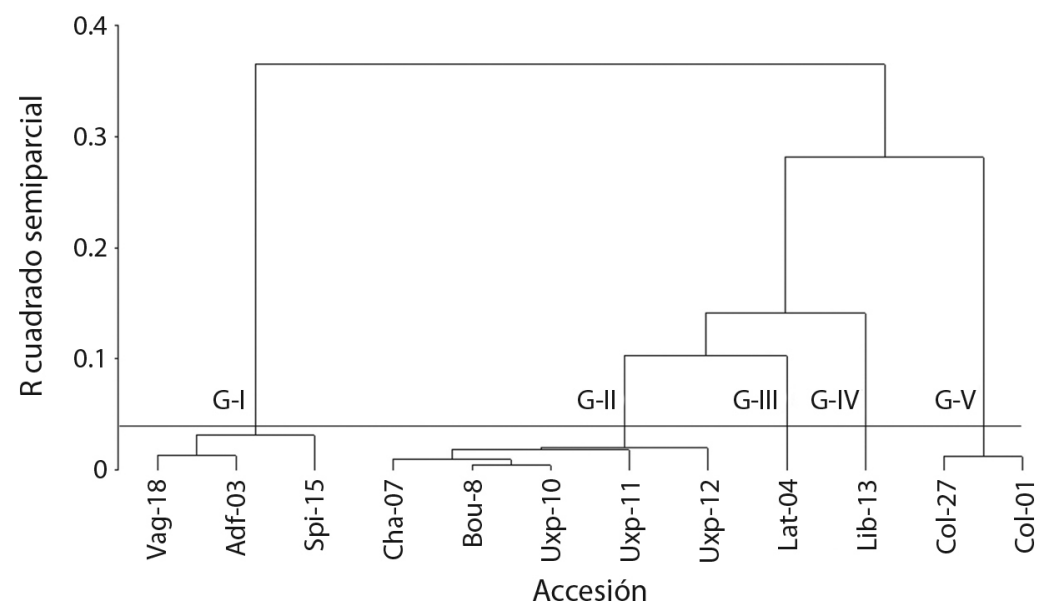

Fig. 5. Dendrograma de 12 accesiones (taxa) de Heliconia en función de diez caracteres morfológicas de polen $\left(\mathrm{R}^{2}=0.05\right)$.

Fig. 5. Dendrogram of 12 Heliconia accessions (taxa) as a function of ten pollen morphological characters $\left(\mathrm{R}^{2}=0.05\right)$.

que tienen el polen morfológicamente similar, ya que presentan una cara distal altamente convexa y con el poro germinativo protudente. Además, presentan un "círculo acanalado" como un aparente engrosamiento en forma de anillo alrededor de la región ecuatorial.

Referente a la morfometría intraespecífica, las tres accesiones de $H$. uxpanapensis comparten valores similares en el eje ecuatorial, aunque existe mayor variación en la forma general del polen debido a la variada convexidad de sus caras distal y proximal. No obstante, las tres accesiones presentan la cara distal convexa, la diferencia más evidente la presentan Uxp-11 y Uxp-12, las cuales tienen una concavidad en la cara proximal. En la ornamentación, Uxp-11 se diferencia por ser verrugosa en su polo distal, mientras que en el polo proximal de Uxp-10 es psilada (Tabla 2).

Heliconia collinsiana, por su parte, tiene el polen homogéneo en todas las variables a excepción de la longitud del eje ecuatorial, donde la var. velutina es casi diez micrómetros menos que el de la var. collinsiana (Tabla 2). En función de dicha diferencia la var. collinsiana, tiene el polen de forma suboblataesferoidal, mientras que la var. velutina tiene forma oblato-esferoidal, lo que significa que la relación $\mathrm{P} / \mathrm{E}$ es cercana a 1.0.

\section{DISCUSIÓN}

El análisis palinológico de las especies del género Heliconia del Neotrópico mexicano, con base en disimilitudes del tipo del poro germinativo, la forma de la cara proximal y distal, mostró que fue posible generar cinco grupos. Por otra parte, con características asociadas a la superficie de la exina fue posible diferenciar especies y variedades dentro de dichos grupos. Resultados similares encontraron Kress \& Stone (1983) en su estudio de 27 especies de Heliconia donde la polaridad, el tipo de poro germinativo, la forma de las caras distal y proximal, permitieron generar cuatro grandes grupos taxonómicos. Por su parte, Ciciarelli y Rolleri (2008) y Ciciarelli, Passarelli y Rolleri (2010), en Canna (Zingiberales), encontraron variación en el tamaño del polen y morfología de las espinas (forma, densidad y su superficie), a pesar que Canna es un género con alta homogeneidad en la forma del polen y el tamaño de sus espinas.

Respecto al tamaño, el polen es grande con base en la clasificación de Sáenz (2004), y los $50.2 \mu \mathrm{m}$ de $H$. collinsiana var. velutina a 70.6 $\mu \mathrm{m}$ de $H$. vaginalis tienen relativa coincidencia con el rango $52 \mu \mathrm{m}$ a $91.3 \mu \mathrm{m}$ que reportaron Kress y Stone (1983) en Heliconia. Asimismo, las especies del presente estudio se limitaron a 
solo polen heteropolar, en contraste con polen heteropolar a isopolar que reportaron Kress y Stone (1982, 1983) y Simão et al. (2007) en especies de Centroamérica y Sudamérica, respectivamente.

La forma convexa de la cara proximal, la alta densidad de microespinas y la presencia de microporos en el téctum agruparon a $H$. vaginalis, $H$. adflexa y $H$. spissa en el grupo I. La alta convexidad en forma de "medio círculo" de la cara proximal de las especies de este grupo, así como de H. librata (Grupo VI), presentan cierta similitud con otras especies como H. talamancana y H. maculata (Kress \& Stone 1983), así como con H. papuana y H. laufao (Kress 1990) de polen esferoidal.

La presencia de microporos en el téctum solamente la reportaron en $H$. angusta (Buchner \& Halbritter, 2010), H. psittacorum y $H$. brasiliensis (Passarelli, Dedominici, \& Rolleri, 2016). Lo anterior obedece a que, aunque existen trabajos palinológicos sobre Heliconia, no hay reportes frecuentes de esta característica, por tanto, son limitadas las especies que la presentan. Es este sentido, con el presente trabajo se amplía el conocimiento sobre el polen de Heliconia, pero, sobre todo, en especies de una zona geográfica que se ha estudiado mínimamente.

El agrupamiento de especies con microporos en el téctum podría indicar alta autoincompatibilidad como lo afirmaron Stone, Sellers y Kress (1979). Los poros de la exina son reservorio de proteínas que intervienen en el proceso de incompatibilidad durante la polinización y la germinación del polen, y esto podría ser congruente con la autoincompatibilidad que se reportaron en especies de Heliconia (Kress, 1983; Meléndez, Rojas, \& Planas, 2008). Sin embargo, es necesario corroborar este supuesto con estudios complementarios a fin de demostrar la supuesta autoincompatibilidad de estas especies si se plantea involucrarlas en trabajos de autopolinización.

Respecto al grupo II, $H$. bourgaeana y $H$. champneiana son especies que presentan similitudes morfológicas difíciles de separar en su fase esporófita (Andersson, 1981), mientras que $H$. uxpanapensis presenta altas similitudes en la inflorescencia con $H$. champneiana (Avendaño et al., 2017), aun cuando H. uxpanapensis es endémica. El agrupamiento de estas tres especies muestra que existe cierta interrelación; sin embargo, trabajos más particulares a nivel de exina e intina podrían dilucidar la filogenia entre estas tres especies que cohabitan en la zona del Neotrópico mexicano.

La ornamentación verrugosa de las caras distal y proximal de H. librata (grupo IV) la hace diferente a las especies estudiadas. Escasas especies reportadas presentan esta característica; no obstante, Simão et al. (2007), en su estudio sobre el polen de $H$. subulata subsp. gracilis (Petersen) L. Andersson y H. velloziana Emygdio, reportaron imágenes del polen con ornamentación de la exina verrugosa, pero la forma es diferente al de H. librata.

Respecto a $H$. collinsiana, por su poro germinativo distinto-protudente representa el grupo V. Las dos variedades de $H$. collinsiana del presente estudio coinciden con las descripciones de las mismas variedades que reportaron Kress y Stone (1983). Sin embargo, el "círculo acanalado" de la vista ecuatorial es un rasgo distintivo de las variedades de $H$. collinsiana de México, rasgo similar al de Heliconia solomonensis Kress, Heliconia lanata (P. Grees) Kress, endémicas del Pacífico (Kress 1990), y a H. angusta Vell (Simão et al., 2007).

Con base en las variables estudiadas, podemos sugerir que el polen de las especies del presente estudio tiene coincidencias con dos de cuatro grupos taxonómicos que Kress y Stone (1983) propusieron para las especies de Centro y Sudamérica. Así, los grupos de poro germinativo distintivo protudente corresponden al grupo nutans mientras que los de polen con poro germinativo indistinto trichocarpha. Cabe mencionar que cada especie mostró particularidades que permiten distinguirlas a nivel de especie y variedad como en $H$. collinsiana.

Los datos morfológicos de Heliconiaceae (monotípica) del presente estudio, así como los que reportaron Kress (1990), Simão et al. (2007), Buchner y Halbritter (2010) y Passarelli et al. (2016), muestran cierta homogeneidad 
de caracteres. Por lo tanto, se puede sugerir que esta familia presenta indicios de polen estenopolínico, con énfasis en algunos grupos detectados, por ejemplo, el grupo de especies de la Fig. 1 (Vag-18, Adf-03 y Spi-15) y Fig. 2 (Cha-07, Bou-08, Uxp-10, Uxp-11 y Uxp12). Sin embargo, aunque se forman grupos polínicos con cierta homogeneidad, es parcial si se les compara a las familias estenopolínicas Gramineae (Perveen, 2006); Malvaceae, Grewioideae (Mambrín, Avanza, \& Ferrucci, 2010) y Chenopodiaceae (Perveen \& Qaiser, 2012) las cuales presentan características altamente homogéneas en todos sus grupos. Más estudios en Heliconiaceae podrían reafirmar la homogeneidad en los grupos polínicos, puesto que las especies hasta ahora estudiadas representan aproximadamente el $23 \%$ de las especies reportadas.

Por otra parte, referente a la morfología intraespecífica, considerando un $\mathrm{R}^{2}$ semiparcial $=0.05$, la variación que se observó en $H$. uxpanapnesis fue en distintas variables y en diferente magnitud. Esta situación la reportaron Kress y Stone (1983) y Tekşen, Aytaç y Pinar (2010), en Fritillaria L. (Liliaceae). Caso contrario a lo que se observó en $H$. collinsiana, donde la var. velutina se diferenció de la var. collinsiana únicamente por la longitud de su eje ecuatorial, al menos para las variables que aquí se estudiaron. Sin embargo, esta característica es altamente consistente y es mínimamente afectada por factores ambientales, debido a que los $50.2 \mu \mathrm{m}$ de $H$. collinsiana var. velutina versus $52 \mu \mathrm{m}$ que reportaron Kress y Stone (1983) demuestra que en longitud ambas son altamente similares. La similitud del polen entre estas dos variedades permite ver que, aunque no son el mismo genotipo puede asegurarse que son la misma variedad botánica debido a que ambas tienen como origen la Sierra Madre Occidental en la vertiente del Pacífico, zona que comparten Guatemala y Chiapas, México. Esto concuerda con lo que encontraron Ciciarelli, Passarelli, \& Rolleri (2010) en Canna, al reportar que la longitud del eje ecuatorial es una variable consistente que permite inclusive diferenciar a las variedades dentro de especies.
La variación intraespecífica a nivel de ornamentación la han reportado también en otros géneros, por ejemplo, en Quercus (Panahi, Pourmajidian, Fallah, \& Pourhashemi, 2012) y en Fritillaria L. (Liliaceae; Tekşen et al., 2010).

Declaración de ética: los autores declaran que todos están de acuerdo con esta publicación y que han hecho aportes que justifican su autoría; que no hay conflicto de interés de ningún tipo; y que han cumplido con todos los requisitos y procedimientos éticos y legales pertinentes. Todas las fuentes de financiamiento se detallan plena y claramente en la sección de agradecimientos. El respectivo documento legal firmado se encuentra en los archivos de la revista.

\section{AGRADECIMIENTOS}

Los autores agradecen al Instituto Nacional de Investigaciones Forestales Agrícolas y Pecuarias por el financiamiento del presente estudio mediante el proyecto No. 1711533202. A María Guadalupe Nieto López por la orientación en la preparación del material biológico previo a la acetólisis.

\section{RESUMEN}

Introducción: El conocimiento palinológico en Heliconia, es básicamente sobre especies de Centroamérica y Sudamérica, mientras que en especies del Neotrópico mexicano los estudios son limitados. Objetivo: Contribuir al conocimiento palinológico y conocer la interrelación morfológica del polen del género Heliconia del Neotrópico mexicano, como parte de los estudios de biología reproductiva que puedan contribuir en futuros trabajos de mejoramiento genético. Métodos: El estudio se realizó en nueve especies (12 accesiones) de Heliconia nativas de México y que se encuentran establecidas en el Banco de Germoplasma del Instituto Nacional de Investigaciones Forestales Agrícolas y Pecuarias (INIFAP). Se recolectó polen de flores en antesis y se conservó durante $24 \mathrm{~h}$ en glutaraldehído al $2 \%$ y etanol al $50 \%$, en seguida se lavó en etanol al $50 \%$ y deshidrató en alcohol al 70, 90 y $100 \%$ por $30 \mathrm{~min} \mathrm{c/u}$. Las muestras se secaron por punto crítico con $\mathrm{CO}_{2}$, se montaron en bases de cilindros de aluminio cubiertas con cinta de carbón conductivo doble adhesiva y, se analizaron con una capa de aproximadamente $20 \mathrm{~nm}$ de oro-paladio. Las muestras se observaron en MEB modelo 
TOPCON, SM-510. Resultados: El polen mide de 50.22 a $70.56 \mu \mathrm{m}$, es heteropolar y es de forma euoblato, suboblato u oblato-esferoidal. Las caras distal y proximal presentan diferentes grados de convexidad; ambas con ornamentación microequinada o microverrugosa. Las características morfológicas, forma del polen, forma de las caras proximal y distal, así como la ornamentación, muestran cierta homogeneidad. Sin embargo, mediante el análisis de agrupamiento jerárquico $\left(\mathrm{R}^{2}=0.05\right)$ se formaron cinco grupos. El grupo I presenta microperforaciones en el téctum, alta densidad de microespinas y alta convexidad de la cara proximal; el grupo II, contrario al grupo I, tienen la cara distal convexa con densidad de microespinas media y la cara proximal plana a subconvexa. El grupo III lo representa $H$. latispatha y el grupo IV H. librata, los dos grupos son de polen pequeño (para el presente estudio). Aunque H. latispatha tiene cara distal con verrugas, la cara proximal plana y poro germinativo distinto-protudente, $H$. librata tiene verrugas en ambas caras. En el grupo V se agrupa $H$. collinsiana, la cual tiene poro germinativo distinto-protudente y una forma de "círculo acanalado" en la cara proximal. Entre variedades, $H$. collinsiana var. collinsiana es $10 \mu \mathrm{m}$ superior a $H$. collinsiana var. velutina en su eje ecuatorial. Conclusiones: La heterogeneidad de caracteres permitió generar grupos polínicos mientras que las características relativamente homogéneas permitieron reconocer especies y variedades dentro de los grupos. La morfología de las especies de Heliconia de México se ajusta a los caracteres previamente descritos; sin embargo, resaltan características adicionales como la presencia de microporos y la forma de "círculo acanalado" en la cara proximal.

Palabras clave: palinología; Heliconia; endémica; diversidad; neotropical.

\section{REFERENCIAS}

Andersson, L. (1981). Revision of Heliconia sect. Heliconia (Musaceae). Nordic Journal of Botany, 1, 759-786.

Avendaño-Arrazate, C.H., Arrazate-Argueta, J.A., OrtizCuriel, S., Moreno-Pérez, E., Iracheta-Donjuan, L., Cortés-Cruz, M. (2017). Morphological Characterization in Wild Species of Heliconias (Heliconia spp) in Mexico. American Journal of Plant Sciences, 8, 1210-1223.

Berry, F., \& Kress, W.J. (1991). Heliconia: An Identification Guide. Smithsonian Institution Press, Washington, D.C

Betts, M.G., Hadley, A.S., \& Kress, W.J. (2015). Pollinator recognition by a keystone tropical plant. Proceedings of the National Academy of Sciences, 112, 3433-3438.

Bruna, E.M., Kress, W.J., Marques, F., \& da Silva, O.F. (2004). Heliconia acuminata reproductive success is independent of local floral density. Acta Amazonica, $34,467-471$.

Buchner, R., \& Halbritter, H. (2010). Heliconia angusta. In R. Buchner \& M. Weber (Eds.), PalDat-A palynological database. Descriptions, illustrations, identification, and information retrieval. PalDat - A palynological database. Retrieved from https://www. paldat.org/pub/Heliconia_angusta/103303

Ciciarelli, M.M., \& Rolleri, C.H. (2008). Morfología, taxonomía y caracterización de siete especies Neotropicales del género (Canna (Cannaceae, Zingiberales). Botanica Complutensis, 32, 157-184.

Ciciarelli, M.M., Passarelli, L.M., \& Rolleri, C.H. (2010). Morfología del polen en especies de Canna (Cannaceae) y su implicancia sistemática. Biología Tropical, $58,63-79$.

Collins, T.J. (2007). ImageJ for microscopy. BioTechniques, 43, 25-30.

Ferreira de Castro, C.E., May, A., \& Gonçalves, C. (2007). Atualização da nomenclatura de espécies do gênero Heliconia (Heliconiaceae). Artigo de revisão. Revista Brasileira de Horticultura Ornamental, 13, 38-62.

Gutiérrez-Báez, C. (1987). Notas del herbario Xal. VIII. Una nueva especie de Heliconia (Heliconiaceae) de México. Biotica, 12, 149-152.

Gutiérrez-Báez, C., Avendaño-Reyes, S., \& Zamora-Crescencio, P. (2016). Heliconia veracruzensis, nueva especie de Veracruz, México. Bouteloua, 25, 101-103.

Halbritter, H., Ulrich, S., Grímsson, F., Weber, M., Zetter, R., Hesse, M., ... Frosch-Radivo, A. (2018). Ilustrated Pollen Terminology ( $2^{\text {nd }}$ Ed.). Cham, Switzerland: Springer International Publishing AG.

Kress, W.J. (1983). Self-incompatibility systems in Central American Heliconia. Evolution, 37, 735-744.

Kress, W.J. (1990). Taxonomy of Old World Heliconia (Heliconiaceae). Allertonia, 6, 1-58.

Kress, W.J., Betancur, J., \& Echeverry, B. (1999). Heliconias-Llamaradas de la Selva Colombiana. Bogotá, Colombia: Cristina Uribe Editores.

Kress, W.J., \& Stone, D.E. (1982). Nature of the sporoderm in monocotyledons, with special reference to the pollen grains of Canna and Heliconia. Grana, 21, 129-148.

Kress, W.J., \& Stone, D.E. (1983). Morphology and phylogenetic significance of exine-less pollen of Heliconia (Heliconiaceae). Systematic Botany, 8, 149-167.

Mambrín, M.V., Avanza, M.M., \& Ferrucci, M.S. (2010). Análisis morfológico y morfométrico del polen de Corchorus, Heliocarpus, Luehea, Mollia y Triumfetta 
(Malvaceae, Grewioideae) en la región austral de América del Sur. Darwiniana, 48, 45-58.

Márquez-Guzmán, J., Wong, R., Pérez, M., López, L., \& Munguía, G. (2016). Técnicas de laboratorio para el estudio del desarrollo en angiospermas. Ciudad de México, México: Universidad Autónoma de México.

Meléndez, A.E., Rojas, S.J., \& Planas, S. (2008). Self-compatibility of microgametophytes in Heliconia bihai (Heliconiaceae) from St. Lucia. Caribbean Journal of Science, 44, 145-149.

Ortiz-Curiel, S., Avendaño-Arrazate, C.H., Olivera de los Santos, A., Grajales-Solís, M., Canul-Ku, J., CortésCruz, M., \& Iracheta-Donjuan, L. (2015). Heliconia L.: Género subutilizado en México. Agroproductividad, 8, 51-59.

Panahi, P., Pourmajidian, M.R., Fallah, A., \& Pourhashemi, M. (2012). Pollen morphology of Quercus (subgenus Quercus, section Quercus) in Iran and its systematic implication. Acta Societatis Botanicorum Poloniae, $81,33-41$.

Passarelli, L.M., Dedominici, A.C., \& Rolleri, C.H. (2016) Polen de Zingiberales de humedales rioplatenses y selvas subtropicales del NE de la Argentina. Botanica Complutensis, 40, 147-160.

Perveen, A. (2006). A contribution to the pollen morphology of family Gramineae. World Applied Sciences Journal, 1, 60-65.
Perveen, A., \& Qaiser, M. (2012). Pollen flora of PakistanLXX: Chenopodiaceae. Pakistan Journal of. Botany, $44,1325-1333$.

Sáenz, L.C. (2004). Glosario de términos palinológicos. Lazaroa, 25, 93-112.

SAS Institute Inc. (2012). SAS® Deployment Wizard and SAS® Deployment Manager 9.3: User's Guide. Cary, NC.

Sheela, V.L., Geetha-Lekshmi, P.R., Jayachandran-Nair, C.S., \& Rajmohan, K. (2006). Molecular characterization of Heliconia by RAPD assay. Journal of Tropical Agriculture, 44, 37-41.

Simão, D.G., Scatena, V.L., \& Bouman, F. (2007). Anther development, microsporogenesis and microgametogenesis in Heliconia (Heliconiaceae, Zingiberales). Flora, 202, 148-160.

Stone, D.E., Sellers, S.C., \& Kress, W.J. (1979). Ontogeny of exineless pollen in Heliconia, a banana relative. Annals of the Missouri Botanical Garden, 66, 701-730.

Suárez-Montes, P., Fornoni, J., \& Nunez-Farfán, J. (2011). Conservation genetics of the endemic mexican Heliconia uxpanapensis in the los tuxtlas tropical rain forest. Biotropica, 43, 114-121.

Tekşen, M., Aytaç, Z., \& Pinar, N.M. (2010). Pollen morphology of the genus Fritillaria L. (Liliaceae) in Turkey. Turkish Journal of Botany, 34, 397-416. 